\title{
Crescimento e Idade de Espécies Nativas Regenerantes Sob Plantio de Araucaria angustifolia no Paraná
}

\author{
Afonso Figueiredo Filho ${ }^{1}$, Franciéle de Souza Retslaff ${ }^{1}$, \\ Fabiane de Souza Retslaff ${ }^{1}$, Tomaz Longhi-Santos ${ }^{2}$, Thiago Floriani Stepka ${ }^{1}$
}

${ }^{1}$ Departamento de Engenharia Florestal, Universidade Estadual do Centro-Oeste - UNICENTRO, Irati/PR, Brasil ${ }^{2}$ Departamento de Engenharia Florestal, Universidade Federal do Paraná - UFPR, Curitiba/PR, Brasil

\begin{abstract}
RESUMO
Este trabalho teve como objetivo usar técnicas dendrocronológicas para recuperar o crescimento diamétrico e a idade média de espécies arbóreas estabelecidas sob um povoamento de Araucaria angustifolia implantado em 1949 na Floresta Nacional de Irati, Paraná. Foram amostradas 30 árvores da espécie regenerante Araucaria angustifolia, 24 da espécie regenerante Cedrela fissilis, 24 da espécie regenerante Clethra scabra, 22 da espécie regenerante Ilex paraguariensis e 18 da espécie regenerante Ocotea puberula. O modelo de Chapman-Richards foi utilizado para descrever as curvas de crescimento em diâmetro por espécie, construindo-se curvas de incremento médio anual (IMA) e incremento corrente anual (ICA). As espécies estudadas apresentaram grande potencial para estudos dendrocronológicos e o uso dessa técnica permitiu determinar que a regeneração natural iniciou-se em 1958 e que tinha, em média, 34 anos. A técnica possibilitou também recompor o crescimento em diâmetro por espécie, gerando taxas de crescimento anuais, com as quais se identificaram os anos característicos de alto e baixo crescimento.
\end{abstract}

Palavras-chave: dendrocronologia, regeneração natural, floresta com Araucária, crescimento e produção.

\section{Growth and Ages of Regenerating Native Species Under Plantation of Araucaria angustifolia in Parana State}

\begin{abstract}
This study aimed to use dendrocronology techniques to recover the diameter growth and the average age of tree species established under an Araucaria angustifolia stand, planted in 1949 in the Irati National Forest, Paraná state. We obtained samples of 30 regenerating trees of the species Araucaria angustifolia, 24 regenerating trees of the species Cedrela fissilis, 24 regenerating trees of the species Clethra scabra, 22 regenerating trees of the species Ilex paraguariensis and 18 regenerating trees of the species Ocotea puberula. The Chapman-Richards model was used to describe the growth curves in diameter by species and curves of mean annual increment (MAI) and current annual increment (CAI) were constructed. Species showed a great potential for dendrochronological studies and the use of this technique allowed determining that natural regeneration began in 1958 and it had an average of 34 years old. The technique also allowed restore growth by species, generating annual growth rates, which identified the characteristic years of high and low growth.
\end{abstract}

Keywords: dendrochronology, natural regeneration, Araucaria forest, growth and yield. 


\section{INTRODUÇÃO}

Uma das maneiras de se avaliar o crescimento e a produtividade florestal é por meio de inventários contínuos com instalação de parcelas permanentes. Todavia, o procedimento necessita de médio e longo prazo para a geração de dados. Nesse aspecto, quando a espécie apresenta anéis de crescimento anuais, a dendrocronologia permite recompor o crescimento de uma árvore de maneira relativamente rápida e confiável. Esse é um dos métodos científicos bastante utilizados atualmente na análise e interpretação do crescimento anual, possibilitando a recuperação de informações sobre a vida do vegetal e permitindo reconstruir fenômenos ecológicos e ambientais ocorridos no passado.

O conhecimento da idade das árvores e de informações que podem ser inferidas do estudo de seus anéis de crescimento são importantes para definição do uso da floresta. O estudo sobre o passado das florestas pode fornecer informações indispensáveis sobre como utilizar, manejar e preservar esse recurso para o futuro (Botosso \& Mattos, 2002). Informações a respeito da dinâmica de crescimento e incremento de espécies arbóreas nativas refletem a principal interação árvore versus ambiente versus espécie, que pode variar com a região (Mattos et al., 2011).

Encinas \& Pinto (2005) descreveram alguns métodos de determinação de idade, tais como a Determinação por Observação, na qual se pode estimar a idade de uma árvore pelo seu tamanho ou aparência geral, com uma simples análise visual. Esse método requer muita experiência e prática, além de o mensurador estar completamente familiarizado com o comportamento silvicultural da espécie e o ambiente em que a árvore está se desenvolvendo. O tamanho e a forma das árvores são afetados diretamente pelas características do sítio e pelas condições em que estão se desenvolvendo, assim, árvores da mesma espécie e com a mesma idade podem apresentar diferenças marcantes nas suas variáveis dendrométricas, o que dificulta a aplicação desse método.

A contagem dos verticilos, que consiste em uma associação entre o número de verticilos e a idade do indivíduo, em anos, é outro método aplicado à determinação da idade, porém essa técnica restringe-se a algumas espécies florestais, como algumas coníferas, nas quais o número de verticilos ao longo do tronco pode corresponder à idade da árvore (Encinas \& Pinto, 2005).

A análise de tronco completa é uma das técnicas mais acuradas para se determinar a idade e também uma das mais utilizadas. Possibilita a recuperação da idade e também do crescimento passado de diâmetro, altura, volume e forma dos troncos das árvores. Pode ser aplicado em qualquer época do ano, mas é aplicada somente em espécies que têm como característica a distinção dos anéis de crescimento anuais. Um outro fator que dificulta seu uso é a necessidade de a árvore ser derrubada, sendo portanto, um método destrutivo. Para contornar esse problema, tem-se disponível o Trado de Pressler, com o qual se extraem rolos de incremento, não sendo necessário derrubar a árvore. Essa técnica é denominada de análise de tronco parcial, apresentando vantagens e desvantagens em relação à análise de tronco completa. Suas principais vantagens são a rapidez na amostragem e ser um método não destrutivo. Tem sido intensivamente utilizado em florestas nativas.

O Brasil é conhecido mundialmente pela sua ampla diversidade de espécies florestais, porém poucos são os estudos e as informações sobre a idade de árvores em condições naturais. São raros os estudos no país sobre a formação dos anéis de crescimento e a determinação da idade em árvores tropicais e subtropicais (Botosso \& Mattos, 2002). Pesquisas sobre a idade e o crescimento de diâmetro, altura e volume de árvores geram inúmeras informações, que podem ser utilizadas na determinação dos ciclos de corte, do regime de desbastes e da estimativa dos cortes e dos volumes admissíveis para uma exploração sustentável. Para a Ecologia, esses aspectos são básicos para os estudos de dinâmica de população, desenvolvimento e produtividade de ecossistemas (Botosso \& Mattos, 2002). A idade de florestas nativas é uma variável difícil de ser mensurada devido à grande heterogeneidade entre as espécies. O manejo e a conservação de florestas nativas dependem de sua regeneração natural, assim, conhecer essa característica é fundamental na proposição de ações de recuperação das populações (Mattos et al., 2011).

Estudos sobre a regeneração natural permitem a realização de previsões sobre o comportamento e desenvolvimento futuro da floresta, pois fornecem a relação e a quantidade de espécies que constituem o 
seu estoque, bem como suas dimensões e distribuição na área (Carvalho, 1982).

No Brasil, plantações florestais com idade avançada em geral apresentam uma quantidade de árvores remanescentes em torno de 300-400 árvores por hectare. Isso possibilita a regeneração espontânea de espécies nativas presentes no banco de sementes ou nas sementes trazidas por diversos meios de dispersão.

Para Carneiro e Rodrigues (2007 apud Viani et al., 2010), há uma tendência de regeneração natural sob plantios apresentar distribuição espacial agregada devido à dispersão das sementes de algumas espécies (barocórica, autocóricas e zoocóricas com dispersores ausentes) concentradas ao redor dos parentais; à preferência das espécies por sítios com melhores níveis de fertilidade do solo; e/ou à disponibilidade de água e de aberturas de clareiras nos plantios, que propiciam a concentração da regeneração, sobretudo de indivíduos de espécies pioneiras.

Na Floresta Nacional de Irati há plantios antigos de Araucaria angustifolia (Bertol.) O. Kuntze das décadas de 1940-1950. No plantio onde esta pesquisa foi realizada, após vários desbastes, existia cerca de 300 árvores ha $^{-1}$, apresentando aparência de floresta nativa devido à grande regeneração espontânea desencadeada em algum momento pelas aberturas dos desbastes realizados (Rode et al., 2010).

Assim, esta pesquisa teve como objetivo usar técnicas dendrocronológicas para avaliar a idade da regeneração espontânea e o crescimento diamétrico de algumas espécies dessa regeneração em um plantio de araucária com 63 anos de idade.

\section{MATERIAL E MÉTODOS}

\section{1. Área de estudo}

Os dados foram coletados na regeneração natural sob um plantio de Araucaria angustifolia na Floresta Nacional de Irati (Flona de Irati) com idade de 63 anos (plantio em 1949, amostragem realizada em 2012). Originariamente, o local era ocupado por Floresta Ombrófila Mista, substituída pelo plantio de araucária. O clima da região, baseando-se na classificação de Köppen, é do tipo $\mathrm{Cfb}$ - Subtropical Úmido Mesotérmico caracterizado por verões frescos, geadas severas e frequentes e sem estação seca definida.
As árvores amostradas foram coletadas em 10 parcelas permanentes com dimensões de $100 \mathrm{~m} \times 100 \mathrm{~m}$ (1 ha) instaladas em 2005 e remedidas a cada 3 anos. No plantio ocorreram capinas por um período de 5-7 anos, tendo sido realizados desbastes ao longo dos anos, remanescendo na área cerca de 300 indivíduos/ha da plantação inicial, com aparência de floresta nativa em virtude da grande regeneração natural sob o povoamento (Rode et al., 2010).

\subsection{Amostragem e trabalho de laboratório}

Nos 10 ha amostrados em 2011 considerando-se um limite de inclusão de $10 \mathrm{~cm}$ de diâmetro (DAP) ocorriam 93 espécies, das quais foram selecionadas 5 bastante presentes na regeneração natural e também porque tinham como característica fundamental anéis de crescimento anuais. Dessa forma foram estudadas as espécies Araucaria angustifolia (Bertol.) O. Kuntze (araucária), Cedrela fissilis Vell. (cedro), Clethra scabra Pers. (carne-de-vaca), Ilex paraguariensis A.St-Hil. (erva-mate) e Ocotea puberula (Reich.) Nees (canela-guaicá). Roik (2012) realizou estudos sobre a dinâmica (2002-2011) dessa regeneração natural e encontrou os seguintes valores de importância (VI\%) e número de árvores por hectare (N/ha), para as espécies selecionadas para o presente estudo: A. angustifolia, $\mathrm{VI}=13,12 \%, \mathrm{~N} / \mathrm{ha}=42,32$; I. Paraguariensis, $\mathrm{VI}=6,68 \%, \mathrm{~N} / \mathrm{ha}=52,12 ;$ C. fissilis, $\mathrm{VI}=3,71 \%, \mathrm{~N} / \mathrm{ha}=14,88 ;$ O. Puberula, $\mathrm{VI}=3,27 \%$, $\mathrm{N} / \mathrm{ha}=13,96$; e C. scabra, VI $=1,61 \%, \mathrm{~N} / \mathrm{ha}=4,24$. Esses valores de importância encontrados por Roik (2012) revelam a importância ecológica relativa de cada espécie na comunidade estudada. Assim, foram amostradas, respectivamente, 30, 22, 24, 18 e 24 árvores das espécies, contemplando todas as classes diamétricas (DAP) de cada espécie.

Os dados para esta pesquisa foram coletados no mês de janeiro de 2012, considerando o período de crescimento de uma primavera a outra para completar um ano de idade da árvore (período vegetativo), no qual seu crescimento de lenho inicial ou primaveril e seu crescimento de lenho tardio ou outonal já estão completos. Considerou-se a idade até o ano de 2010, pois, o crescimento do ano 2011 ainda não estava completo.

Em cada árvore selecionada foram retirados dois rolos de incremento com o Trado de Incremento de Pressler a 1,3 $\mathrm{m}$ do solo (DAP), os quais foram colocados em suportes de madeira e fixados com fita adesiva para 
evitar rachaduras nas amostras durante a secagem natural. Após a secagem, os rolos de incremento foram colados nos suportes e, posteriormente, lixados, utilizando-se lixas para madeira com quatro diferentes graduações $\left(80,100,180\right.$ e 320 grãos $\left./ \mathrm{cm}^{2}\right)$, com o objetivo de melhor visualização dos anéis de crescimento.

Para a contagem e medição dos anéis foi utilizado o LINTAB 6 e o software TSAP-Win, com precisão de 0,01 mm, disponíveis no Laboratório de Manejo Florestal da Unicentro, Campus de Irati. Em cada amostra analisada e para todas as espécies foram acrescidos 3 anos (anéis), tempo médio estimado para que árvores das espécies estudadas possam atingir 1,3 m (DAP). Nesse aspecto é importante ressaltar que a tradagem na base (onde se espera ter todos os anéis de crescimento, ou idade total) é de difícil coleta. Além disso, estudos do crescimento em diâmetro, em geral, são tradicionalmente realizados na altura do DAP.

\subsection{Controle de qualidade das séries de anéis de crescimento e estudo dendrocronológico}

A verificação da sincronização foi realizada com o programa COFECHA, que analisa estatisticamente a correlação entre as porções da série flutuante (a datar) e séries individuais ou máster, independentemente datadas e verificadas, possibilitando identificar a existência de falsos anéis de crescimento para seu posterior controle (Alvarado, 2009). As correlações foram calculadas em períodos de 50 anos comparados a cada 25 anos, com um nível de confiança de 99\%.

Com intuito de identificar padrões de crescimento entre as espécies amostradas foi realizada a padronização das cronologias, a qual foi obtida a partir das séries de medidas de largura dos anéis de crescimento das árvores, utilizando-se o programa ARSTAN (Cook \& Krusic, 1985).
A partir da medição dos rolos de incremento de cada árvore foi realizada a contagem dos anéis de crescimento e a medição do crescimento diamétrico anual, construindo-se curvas de crescimento acumulado e curvas de incremento. O incremento corrente anual (ICAd) e o incremento médio anual (IMAd) em diâmetro foram calculados pelas Fórmulas 1 e 2, respectivamente:

$I C A d=d_{i+1}-d_{i}$

$I M A d=d_{i} / n$

em que: $\mathrm{d}_{\mathrm{i}}=$ DAP sem casca na idade $\mathrm{i}(\mathrm{cm}) ; \mathrm{d}_{\mathrm{i}+1}=$ DAP sem casca na idade $\mathrm{i}+1(\mathrm{~cm}) ; \mathrm{n}=$ número de anos.

A fim de padronizar o crescimento médio por espécie, ajustou-se o modelo biológico de Chapman-Richards (Equação 3) aos dados de produção em diâmetro. A primeira derivada do modelo gera a curva de ICA, enquanto que o IMA é a média da curva de produção. O modelo biológico tem a forma:

$d a p=b_{0}\left[\left(1-\exp \left(-b_{1} \cdot I\right)\right)\right]^{b_{2}}$

em que: DAP $=$ diâmetro à altura do peito $(\mathrm{cm})$; $\mathrm{I}=$ idade (anos); $\exp =$ exponencial; $b_{i}=$ coeficientes a serem estimados.

\section{RESULTADOS E DISCUSSÃO}

A execução do programa COFECHA nas séries de largura dos anéis de crescimento permitiu controlar a qualidade das séries, sincronizando-as com a série máster (média das séries individuais), detectando-se os erros de medição e gerando-se os parâmetros indicadores de qualidade das séries resultantes (Tabela 1).

Tabela 1. Resumo dos resultados do controle de qualidade das séries executadas pelo programa COFECHA para as cinco espécies estudadas.

Table 1. Summary of results of quality control of the series performed by COFECHA software for the 5 studied species.

\begin{tabular}{cccccc} 
Parâmetros & A. angustifolia & C. fissilis & I. paraguariensis & C. scabra & O. puberula \\
\hline Período considerado & $1958-2010$ & $1963-2010$ & $1969-2010$ & $1976-2010$ & $1961-2010$ \\
(r) média com a máster & 0,403 & 0,307 & 0,367 & 0,311 & 0,241 \\
(r) séries/máster & $0,129-0,581$ & $0,108-0,433$ & $0,073-0,537$ & $0,145-0,563$ & $0,085-0,467$ \\
Sensibilidade média (s) & 0,305 & 0,365 & 0,302 & 0,298 & 0,328 \\
\hline Autocorrelação & $0,401-0,785$ & $0,454-0,799$ & $0,498-0,750$ & $0,447-0,722$ & $0,561-0,736$ \\
\hline
\end{tabular}

(r): intercorrelação média com a máster; (r) séries/máster: variação da intercorrelação das séries individuais com a máster; valores críticos variaram de 0,3281 a $1 \%$. 
Os resultados obtidos possibilitaram uma avaliação positiva sobre o potencial de estudos dendrocronológicos para as cinco espécies. A datação mostrou trechos com altas e baixas correlações (não significativas com valores abaixo do valor de 0,3281 a $1 \%$ ) para as espécies em estudo, com valores mais altos para a araucária. Stepka (2012), em seus estudos na região de Irati, Paraná, encontrou valores de intercorrelação para a araucária entre 0,33 e 0,5, com uma média de 0,452, valores semelhantes ao desta pesquisa.

$\mathrm{Na}$ determinação da idade da regeneração espontânea, buscou-se não eliminar as amostras, pois, para a determinação da idade média da regeneração natural, faz-se necessário um número representativo de árvores amostradas para o povoamento, o que torna os resultados mais seguros. Para C. fissilis, Stepka (2012) encontrou valores entre 0,203 e 0,689 e uma média de 0,446 , superiores aos valores encontrados neste trabalho. Ferreira (2012) obteve em seus estudos uma correlação média que variou de acordo com o núcleo de estudo de 0,162 a 0,429, valores que se assemelham aos encontrados no presente estudo. Para as espécies C. scabra, I. paraguariensis e $O$. puberula não foram encontrados valores de intercorrelação descritos na literatura. As correlações abaixo do ponto crítico para significância dos dados ( $\mathrm{p}$-valor $\leq 0,01)$ podem advir da dificuldade de se identificar, nos rolos de incremento, os falsos anéis de crescimento ou anéis descontínuos.

Sensibilidade é uma estatística especificamente concebida para analisar as variações na largura do anel e que corresponde à variação média da largura do anel em relação à próxima. Assim, quanto maior a sensibilidade, menor a autocorrelação, o que caracteriza uma maior variância nos dados (Mattos \& Braz, 2012). A sensibilidade está diretamente ligada às condições do sítio, o que pode explicar os valores de sensibilidade encontrados serem semelhantes para as espécies em estudo, exceto para C. scabra, que apresentou o valor de sensibilidade média mais baixo $(0,298)$.

O estudo da idade de uma floresta torna-se um conceito vago, pois nem todas as árvores que a compõem iniciam o seu crescimento ao mesmo tempo. Nesse sentido, adotou-se a idade média das árvores como forma de aproximação. Porém, para as práticas de manejo florestal, faz-se necessário que as florestas nativas e os reflorestamentos possam ser caracterizados por uma idade definida (Encinas \& Pinto, 2005). Assim, na Tabela 2 são apresentados os valores mínimos, máximos e médios para a variável idade determinados nesta pesquisa, utilizando-se todas as árvores amostradas, tendo-se acrescentado 3 anos de idade para cada árvore amostrada, como justificado na metodologia.

Diante dos resultados encontrados, o processo de regeneração natural sob o plantio de araucária teve início por volta de 1958 (Figura 1). Considerando-se que o plantio foi estabelecido no ano de 1949 e que, segundo os relatórios disponibilizados pela administração da Flona, nos primeiros 5 anos do plantio houve intervenção humana por meio de capina e roçadas (atividades de manutenção do plantio), a regeneração espontânea foi iniciada pela própria araucária 9 anos após o plantio, seguido pelas espécies O. puberula, C. fissilis, I. paraguariensis e C. scabra. Assim, as 5 espécies analisadas iniciaram a regeneração natural em um período de 8 a 18 anos (1958 a 1968) após o plantio. Vale ressaltar que na área de estudos, além das araucárias plantadas, existem algumas árvores nativas remanescentes que não foram derrubadas na época do plantio. Também é importante mencionar que ao lado da área de estudos existem remanescentes de Floresta Ombrófila Mista. Isso, sem dúvida, justifica o surgimento de regeneração natural da araucária, após 9 anos da realização do plantio, considerando

Tabela 2. Idades mínima, média e máxima (anos) estimadas para as espécies estudadas.

Table 2. Minimum, average and maximum ages (years) estimated for the studied species.

\begin{tabular}{|cccc}
\hline Espécie & Idade mínima & Idade máxima & Idade média \\
\hline A. angustifolia & 22 & 52 & 34 \\
\hline I. paraguariensis & 19 & 46 & 33 \\
\hline O. puberula & 20 & 50 & 35 \\
C. scabra & 21 & 42 & 32 \\
\hline C. fissilis & 20 & 48 & 35 \\
\hline Idade média & - & - & 33,8 \\
\hline
\end{tabular}


que a produção de pinhões ocorre, em geral, a partir dos 12 anos de idade.

Pode-se, assim, inferir que a regeneração espontânea, com base nessas importantes espécies presentes no plantio, tem aproximadamente 34 anos (idade média de todas as espécies amostradas), ou seja, iniciou por volta de 1958 e, a partir de então, o processo de regeneração desenvolveu condições ambientais para o desenvolvimento das demais espécies encontradas atualmente na área de 10 hectares da Flona. Na Figura 1 mostra-se, esquematicamente, o surgimento das espécies regenerantes na área com base nas árvores amostradas de cada espécie. A primeira árvore oriunda do processo de regeneração natural para a espécie araucária, com base nas 30 árvores amostradas, brotou no ano de 1958.

Para verificar o padrão de crescimento das espécies estudadas foram utilizados os índices de crescimento gerados pelo software ARSTAN, sendo gerados padrões gráficos relacionais entre as várias amostras, criando-se um padrão médio geral (Figura 2).
Os índices de crescimento para as cinco espécies em estudo apontam picos de crescimento, alto ou baixo, no período avaliado. Pode-se observar que em 2000 houve um crescimento expressivo para as espécies A. angustifolia, O. puberula e C. scabra. Entre 1978 e 1980 as espécies A. angustifolia e O. puberula apresentaram baixo crescimento.

A. angustifolia e O. puberula apresentaram ritmos de crescimento semelhantes. Isso também é notado por meio da correlação entre os índices de crescimento apresentados na Tabela 3, na qual essas duas espécies tiveram maior correlação entre seus índices de crescimento, com $\mathrm{r}=0,66$. O. puberula e C. scabra também apresentaram correlações significativas entre seus índices de crescimento, com $\mathrm{r}=0,47$. C. fissilis e I. paraguariensis apresentaram correlação significativa, com $r=0,37$, e, graficamente, apresentam ritmos de crescimento semelhantes.

$\mathrm{Na}$ Tabela 4 são apresentados os coeficientes e as estatísticas de ajuste e precisão do modelo de

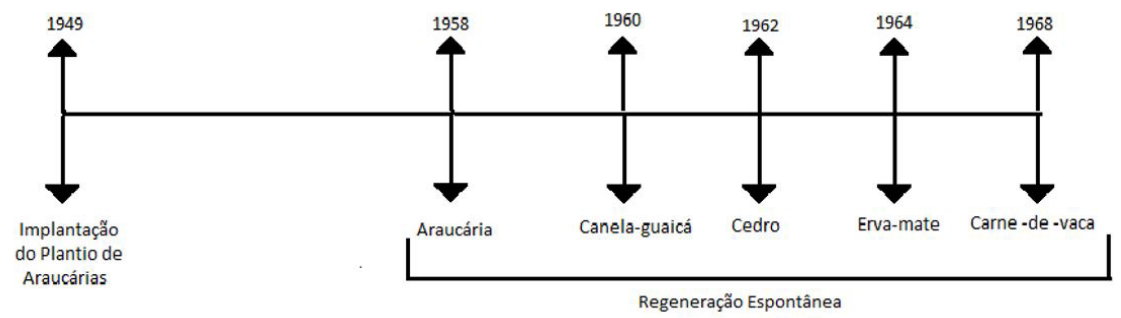

Figura 1. Ano de plantio da araucária e ano de início da regeneração espontânea das espécies estudadas.

Figure 1. Year of planting of Araucaria and year that started the spontaneous regeneration of the each studied species.

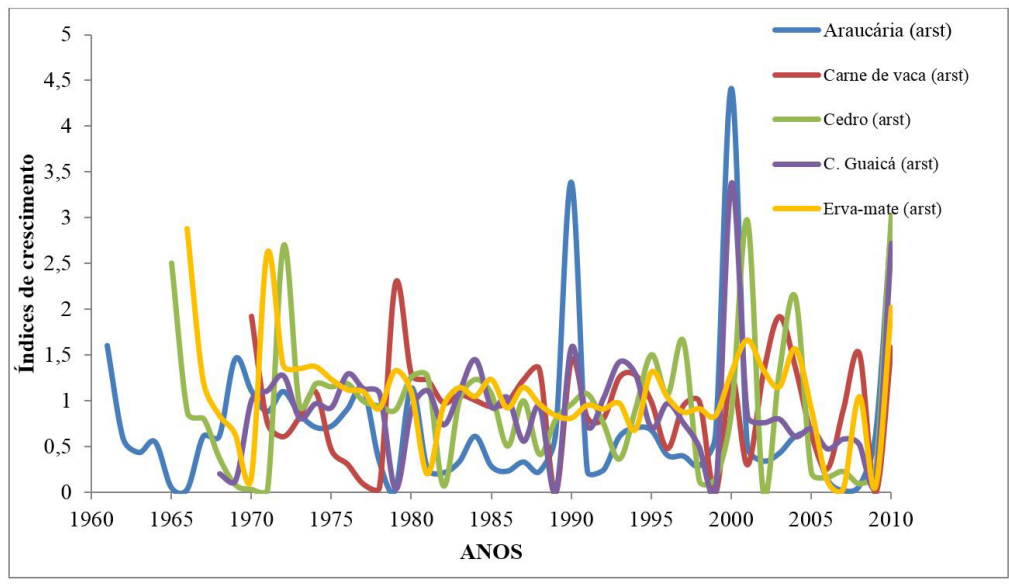

Figura 2. Índices de crescimento gerados pelo ARSTAN para cada espécie.

Figure 2. Growth index generated by ARSTAN for each specie. 
Tabela 3. Correlação dos índices de crescimento para as espécies estudadas.

Table 3. Correlation of growth indices for the studied species.

\begin{tabular}{cccccc} 
Espécie & A. angustifolia & C. fissilis & I. paraguariensis & O. puberula & C. scabra \\
A. angustifolia & 1,0000 & 0,1673 & 0,1226 & 0,6649 & 0,1402 \\
C. fissilis & 0,1673 & 1,0000 & 0,3739 & 0,3625 & 0,1490 \\
I. paraguariensis & 0,1226 & 0,3739 & 1,0000 & 0,3338 & 0,2315 \\
O. puberula & 0,6649 & 0,3625 & 0,3338 & 1,0000 & 0,4753 \\
C. scabra & 0,1402 & 0,1490 & 0,2315 & 0,4753 & 1,0000 \\
\hline
\end{tabular}

Tabela 4. Coeficientes e estatísticas de ajuste e precisão para o modelo de Chapman-Richards para estimar o diâmetro por espécie.

Table 4. Coefficients and statistics of adjustment and precision for the Chapman-Richards model to estimate the diameter by species.

\begin{tabular}{|c|c|c|c|c|}
\hline Espécie & Coeficientes & $\mathbf{R}^{2}$ & Syx $(\mathrm{cm})$ & Syx $\%$ \\
\hline \multirow{3}{*}{ A. angustifolia } & $\mathrm{b} 0=16,79170$ & 0,6769 & 2,61 & 28,13 \\
\hline & $\mathrm{b} 1=0,05361$ & & & \\
\hline & $\mathrm{b} 2=1,24075$ & & & \\
\hline \multirow{3}{*}{ O. puberula } & $\mathrm{b} 0=53,17120$ & 0,7327 & 4,26 & 30,57 \\
\hline & $\mathrm{b} 1=0,01810$ & & & \\
\hline & $\mathrm{b} 2=1,12562$ & & & \\
\hline \multirow{3}{*}{ C. scabra } & $\mathrm{b} 0=19,24340$ & 0,8834 & 1,36 & 17,14 \\
\hline & $\mathrm{b} 1=0,04568$ & & & \\
\hline & $\mathrm{b} 2=1,49441$ & & & \\
\hline \multirow{3}{*}{ C. fissilis } & $\mathrm{b} 0=34,43740$ & 0,7389 & 2,35 & 28,35 \\
\hline & $\mathrm{b} 1=0,01680$ & & & \\
\hline & $\mathrm{b} 2=1,08107$ & & & \\
\hline \multirow{3}{*}{ I. paraguariensis } & $\mathrm{b} 0=20,44830$ & 0,9220 & 1,09 & 15,23 \\
\hline & $\mathrm{b} 1=0,03576$ & & & \\
\hline & $\mathrm{b} 2=1,43892$ & & & \\
\hline
\end{tabular}

$\mathrm{R}^{2}=$ coeficiente de determinação; Syx = erro padrão de estimativa.

Chapman-Richards para estimar o crescimento acumulado em diâmetro (sem casca) para cada uma das cinco espécies estudadas. Observa-se que o melhor ajuste foi obtido para I. paraguariensis, seguido pelo da C. scabra. O coeficiente b0 no modelo de Chapman-Richards indica o valor assintótico para a variável em estudo (DAP). Esse valor foi de $16,79 \mathrm{~cm}$ para A. angustifolia, considerado muito baixo. Isso deve-se ao fato de os dados de DAP utilizados para o ajuste na sua maioria variarem de 8 a $20 \mathrm{~cm}$ sem casca.

Constata-se com base nas 118 árvores amostradas, somando-se as cinco espécies, um incremento médio anual (IMA) de $0,46 \mathrm{~cm}$ sem casca na idade 30 anos e, nessa idade, a espécie com maior IMA anual foi $O$. Puberula, com $0,660 \mathrm{~cm}$, seguindo-se, A. angustifolia, C. fissilis, C. scabra e I. Paraguariensis com, respectivamente, $0,424 \mathrm{~cm} ; 0,422 \mathrm{~cm} ; 0,414 \mathrm{~cm}$; e $0,373 \mathrm{~cm} /$ ano. É relevante mencionar que todas as espécies apresentaram IMA máximo antes dos
30 anos. Na Figura 3 são apresentadas as curvas de produção (crescimento acumulado) e de incrementos (ICA e IMA) em diâmetro ajustadas pelo modelo de Chapman-Richards para as espécies em estudo.

Observa-se que, para $A$. angustifolia, o ponto de encontro entre as curvas de ICA e IMA ocorreu perto dos 8 anos, já para C. scabra esse ponto aconteceu mais tarde, perto dos 16 anos. Para as demais espécies estudadas, o cruzamento das curvas ocorre aos 8, 12 e 19 anos, respectivamente, para C. fissilis, O. puberula e I. paraguariensis (Figura 3).

Stepka (2012), trabalhando com modelagem do crescimento e dendrocronologia em árvores nativas de A. angustifolia, C. fissilis e O. porosa no sul do Brasil, menciona que o ponto de interseção das curvas de ICA e IMA para A. angustifolia foi próximo dos 20 anos em Chapecó, Irati, São Francisco de Paula, São João do Triunfo e Três Barras, e dos 30 anos em Caçador, idades superiores às encontradas neste estudo. 


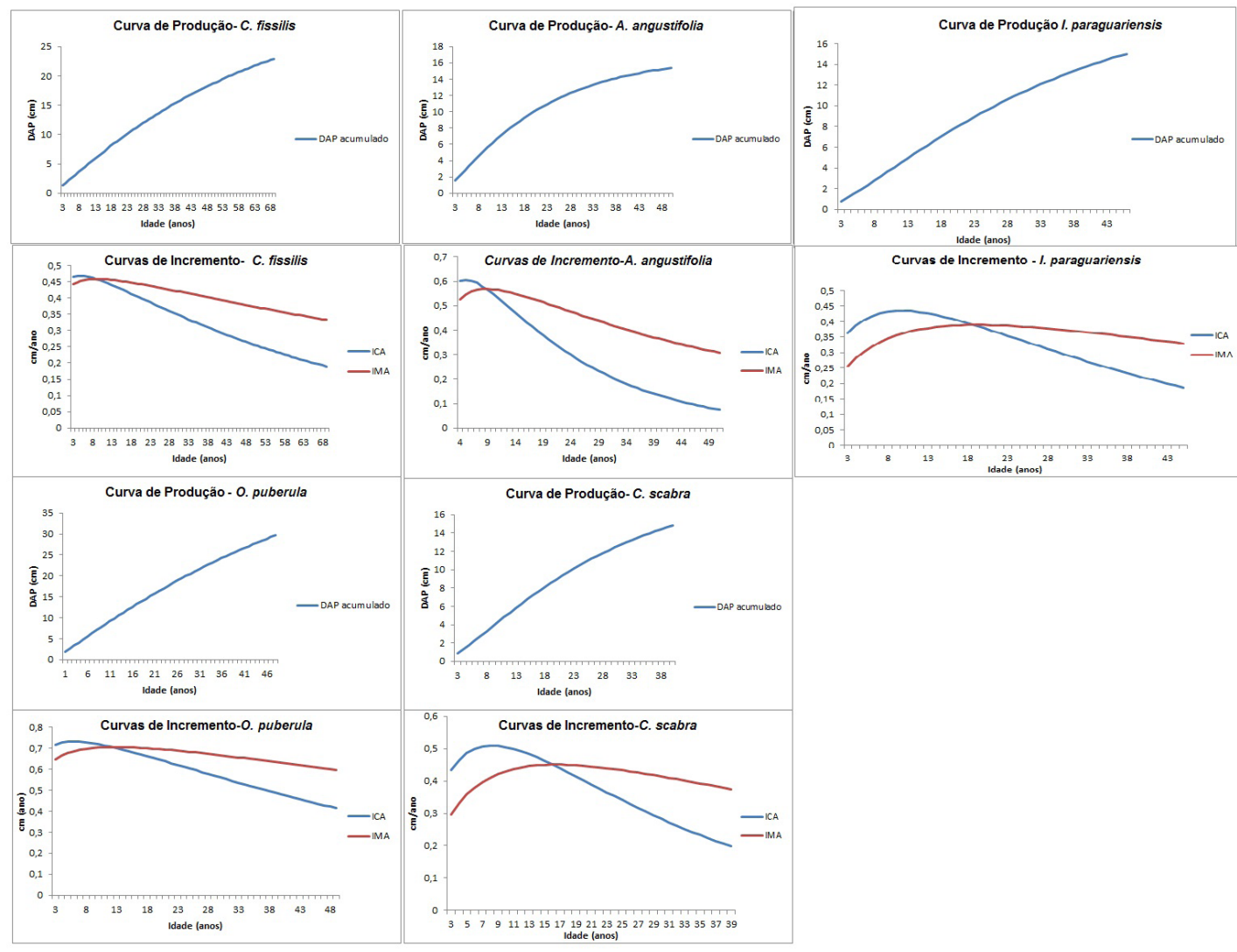

Figura 3. Curvas de produção (crescimento acumulado), incremento corrente anual (ICA) e incremento médio anual (IMA).

Figure 3. Yield (cumulative growth), Current Annual Increment (CAI) and Mean Annual Increment (MAI) curves.

\section{CONCLUSÕES}

- A regeneração espontânea teve início a partir de 1958, 18 anos após a implantação do povoamento de araucária, apresentando as espécies amostradas idade média de 34 anos, sendo que $A$. angustifolia foi a primeira espécie a se regenerar, fruto provavelmente de árvores nativas da espécie que não foram cortadas quando da implantação do plantio.

- Conforme o modelo biológico de Chapman-Richards, as 5 espécies estudadas apresentaram IMA máximo antes dos 19 anos de idade (cruzamento das curvas de ICA e IMA).

- Com base na amostra (118 árvores) englobando as 5 espécies, a taxa de incremento médio anual (IMA) em diâmetro foi de $0,46 \mathrm{~cm}$ sem casca na idade de 30 anos e, nessa idade, a espécie com maior IMA foi $O$. Puberula, com $0,66 \mathrm{~cm}$, seguindo-se A. angustifolia, C. fissilis, C. scabra e I. Paraguariensis com, respectivamente, $0,424 \mathrm{~cm} / \mathrm{ano} ; 0,422 \mathrm{~cm} / \mathrm{ano}$; $0,414 \mathrm{~cm} / \mathrm{ano}$; e $0,373 \mathrm{~cm} / \mathrm{ano}$.

\section{STATUS DA SUBMISSÃO}

Recebido: 15 jul., 2014

Aceito: 26 dez., 2016

\section{AUTOR(ES) PARA CORRESPONDÊNCIA}

\section{Fabiane de Souza Retslaff}

Departamento de Engenharia Florestal, Universidade Estadual do Centro-Oeste UNICENTRO, CEP 84500-000, Irati, PR, Brasil e-mail: faretslaff@gmail.com

\section{REFERÊNCIAS}

Alvarado RJ. Dendrocronologia de árvores de mogno, Swietenia macrophylla King., Meliaceae, ocorrentes na floresta tropical Amazônica do Departamento de Madre de Dios, Peru [dissertação]. São Paulo: Escola Superior 
de Agricultura Luiz de Queiroz, Universidade de São Paulo; 2009.

Botosso PC, Mattos PP. A idade das árvores: importância e aplicação [online]. Colombo: EMBRAPA Florestas; 2002 [citado em 2014 abr 22]. Documentos n. 75. Disponível em: http://ainfo.cnptia.embrapa.br/digital/bitstream/ item/17085/1/doc75.pdf.

Carvalho JOP. Análise estrutural da regeneração natural em floresta tropical densa na região do Tapajós no Estado do Pará [dissertação]. Curitiba: Universidade Federal do Paraná; 1982.

Cook ER, Krusic P. ARSTAN software [online]. New York: Columbia University; 1985 [citado em 2012 out 24]. Disponível em: http://www.ldeo.columbia.edu/treering-laboratory/resources.

Encinas JI, Pinto JRRS. Idade e crescimento das árvores. Comunicações Técnicas Florestais 2005; 7(1): 1-40.

Ferreira GB. Análise dendroclimatológica do cedro (Cedrela fissilis L.-Meliaaceae) para reconstrução do cenário ambiental recente da cidade de São Paulo, SP [dissertação]. Piracicaba: Instituto de Biociências, Universidade de São Paulo; 2012.

Mattos PP, Braz EM. Reunião técnica temática: anéis de crescimento e clima [online]. Colombo: EMBRAPA
Florestas; 2012 [citado em 2013 jun 22]. Disponível em: http: // www.bibliotecaflorestal.ufv.br/ brbitstream/ handle/123456789/5425/Documentos_236.pdf.

Mattos PP, Braz EM, Hess AF, Salis SMA. Dendrocronologia e o manejo florestal sustentável em florestas tropicais [online]. Brasília: Infoteca; 2011 [citado em 2011 nov 22]. Disponível em: www.infoteca.cnptia.embrapa.br/.../ doc/.../Doc218.pdf.

Rode R, Figueiredo A Fo, Machado AS, Galvão F. Análise do padrão espacial de espécies e de grupos florísticos estabelecidos em um povoamento de Araucaria angustifolia e em uma Floresta Ombrófila Mista no centro-sul do Paraná. Floresta 2010; 40(2): 255-268.

Roik M. Dinâmica (2002-2011) e modelagem do incremento diamétrico em fragmento de Floresta Ombrófila Mista na Floresta Nacional de Irati, Paraná [dissertação]. Irati: Universidade Estadual do Centro Oeste; 2012.

Stepka TF. Modelagem do crescimento e dendrocronologia em árvores nativas de Araucaria angustifolia, Cedrela fissilis e Ocotea porosa no sul do Brasil [tese]. Curitiba: Universidade Federal do Paraná; 2012.

Viani RAG, Durigan G, Melo ACG. A regeneração natural sob plantações florestais: desertos verdes ou redutos de biodiversidade? Ciência Florestal 2010; 20(3): 533-552. 